\title{
15.
}

\section{NOTE SUR DEUX FORMULES DONNEES PAR M. M. EISENSTEIN ET HESSE.}

[From the Journal für die reine und angewandte Mathematik, (Crelle) vol. xxIx. (1845), pp. 54-57.]

Mr. Eisenstein a donné [Journal, t. XxVII. (1844), pp. 105-106] cette formule assez remarquable :

$$
\left(a^{2} d^{2}-3 b^{2} c^{2}+4 a c^{3}+4 b d^{3}-6 a b c d\right)^{3}=A^{2} D^{2}-3 B^{2} C^{2}+4 A C^{3}+4 D B^{3}-6 A B C D,
$$

où $A, B, C, D$ sont des fonctions données de $a, b, c, d$. Cela peut se généraliser comme suit.

Soit

$u=a^{2} h^{2}+b^{2} g^{2}+c^{2} f^{2}+d^{2} e^{2}-2 a h b g-2 a h c f-2 a h d e-2 b g c f-2 b g d e-2 c f d e+4 a d f g+4 b c e h$, et de plus

$$
A=\frac{1}{2} \frac{d u}{d a}, \quad B=\frac{1}{2} \frac{d u}{d b}, \quad C=\frac{1}{2} \frac{d u}{d c}, \ldots \quad H=\frac{1}{2} \frac{d u}{d h} .
$$

Représentons par $U$ la même fonction de $A, B, C, \ldots H$, que la fonction $u$ l'est des quantités $a, b, c, \ldots h$, l’on a l'équation

$$
U=u^{3} .
$$

C'est un cas particulier d'une propriété générale de la fonction $u$, que l'on peut énoncer ainsi. Imaginons la fonction

$$
a x_{1} y_{1} z_{1}+b x_{1} y_{1} z_{2}+c x_{1} y_{2} z_{1}+d x_{1} y_{2} z_{2}+e x_{2} y_{1} z_{1}+f x_{2} y_{1} z_{2}+g x_{2} y_{2} z_{1}+h x_{2} y_{2} z_{2}
$$

transformée en

$$
a^{\prime} x_{1}^{\prime} y_{1}^{\prime} z_{1}^{\prime}+b^{\prime} x_{1}^{\prime} y_{1}^{\prime} z_{2}^{\prime}+\ldots \quad+h^{\prime} x_{2}^{\prime} y_{2}^{\prime} z_{2}^{\prime},
$$

C. 
par les substitutions

$$
\begin{array}{lll}
x_{1}=\lambda_{1} x_{1}{ }^{\prime}+\lambda_{2} x_{2}^{\prime}, & y_{1}=\mu_{1} y_{1}^{\prime}+\mu_{2} y_{2}^{\prime}, & z_{1}=\nu_{1} z_{1}^{\prime}+\nu_{2} z_{2}^{\prime}, \\
x_{2}=\lambda_{1}^{\prime} x_{1}^{\prime}+\lambda_{2}^{\prime} x_{2}^{\prime}, & y_{2}=\mu_{1}^{\prime} y_{1}^{\prime}+\mu_{2}^{\prime} y_{2}^{\prime}, & z_{2}=\nu_{1}^{\prime} z_{1}^{\prime}+\nu_{2}^{\prime} z_{2}^{\prime} ;
\end{array}
$$

et soit $u^{\prime}$, la fonction analogue à $u$, des nouveaux ccefficients $a^{\prime}, b^{\prime}, c^{\prime}, \ldots h^{\prime}$ : alors

$$
u^{\prime}=\left(\lambda_{1} \lambda_{2}^{\prime}-\lambda_{2} \lambda_{1}^{\prime}\right)^{2}\left(\mu_{1} \eta_{2}^{\prime}-\mu_{2} \mu_{1}\right)^{2}\left(\nu_{1} \nu_{2}^{\prime}-\nu_{2} \nu_{1}^{\prime}\right)^{2} \cdot u
$$

En échangeant seulement les $z$, ceci donne

$$
u^{\prime}=\left(\nu_{1} \nu_{2}^{\prime}-\nu_{2} \nu_{1}^{\prime}\right)^{2} \cdot u,
$$

ou

$$
\begin{array}{llll}
a^{\prime}=\nu_{1} a+\nu_{1}^{\prime} e, & b^{\prime}=\nu_{1} b+\nu_{1}^{\prime} f, & c^{\prime}=\nu_{1} c+\nu_{1}^{\prime} g, & d^{\prime}=\nu_{1} d+\nu_{1}^{\prime} h, \\
e^{\prime}=\nu_{2} a+\nu_{2}^{\prime} e, & f^{\prime}=\nu_{2} b+\nu_{2}^{\prime} f, & g^{\prime}=\nu_{2} c+\nu_{2}^{\prime} g, & h^{\prime}=\nu_{2} d+\nu_{2}^{\prime} h .
\end{array}
$$

Soit

$$
\begin{aligned}
& \nu_{1}=a h-b g-c f-d e, \\
& \nu_{1}^{\prime}=-2(a d-b c), \\
& \nu_{2}=-2(e h-f g), \\
& \nu_{2}^{\prime}=a h-b g-c f-d e\left(=\nu_{1}\right),
\end{aligned}
$$

on trouve d'abord

$$
\left(\nu_{1} \nu_{2}^{\prime}-\nu_{2} \nu_{2}^{\prime}\right)=u^{2} \text {, ou } u^{\prime}=u^{3},
$$

et puis, en ayant égard aux valeurs de $A, B, C, \ldots H$ :

$$
\begin{aligned}
& a^{\prime}=H, \quad b^{\prime}=-G, \quad c^{\prime}=-F, \quad d^{\prime}=E, \\
& e^{\prime}=D, \quad f^{\prime}=-B, \quad g^{\prime}=-C, \quad h^{\prime}=A,
\end{aligned}
$$

de manière que $u^{\prime}=U$, d'où enfin

$$
U=u^{3} \text {. }
$$

La propriété de la fonction $u$ que je viens d'énoncer, se rapporte à une théorie assez générale, d'une nouvelle espèce de fonctions:algébriques dont je m’occupe actuellement, et lesquelles à cause de leur analogie avec les déterminantes, on pourrait comme je crois nommer "Hyperdéterminantes." Je me propose de poser les premiers fondemens de cette théorie dans un mémoire qui va paraître dans le prochain No. du Cambridge Mathematical Journal (No. xxiII.) [13].

A présent jẹ passe à une formule de Mr. Hesse [Journal, t. xxvIII. (1844), p. 88], qui se rapporte aussi à la même théorie. Soit $V$, une fonction homogène du troisième ordre, et à trois variables $x, y, z$. Soit $\nabla U$, la déterminante formée avec les coefficients du second ordre de $V$, arrangés en cette forme:

$$
\begin{array}{lll}
\frac{d^{2} V}{d x^{2}}, & \frac{d^{2} V}{d x d y}, & \frac{d^{2} V}{d x d z}, \\
\frac{d^{2} V}{d x d y}, & \frac{d^{2} V}{d y^{2}}, & \frac{d^{2} V}{d y d z}, \\
\frac{d^{2} V}{d z d x}, & \frac{d^{2} V}{d z d y}, & \frac{d^{2} V}{d z^{2}} ;
\end{array}
$$


soit $a$ une quantité constante quelconque et soient $A$ et $B$ deux autres constantes déterminés: Mr. Hesse à démontré l'équation remarquable

$$
\nabla(U+a \nabla U)=A U+B \nabla U
$$

mais sans donner la forme des coefficients $A$ et $B$, ce qui parait être très difficile à effectuer. En considérant le cas d'une fonction homogène de deux variables seulement, mais d'ailleurs d'un ordre quelconque, on parvient à un théorème analogue qui m’a paru intéressant.

"Soit $U$ une fonction homogène et de l'ordre $\nu$ des deux variables $x$, $y$, et $\nabla U$ la déterminante $\frac{d^{2} V}{d x^{2}} \cdot \frac{d^{2} V}{d y^{2}}-\left(\frac{d^{2} V}{d x d y}\right)^{2}$, l'on a

$$
\begin{aligned}
(\nu-2)(\nu-3)^{3} \cdot \nabla(U+a \nabla U)=\{-\nu(\nu-1) & \left.(\nu-3)^{2} J a+\nu(\nu-1)(2 \nu-5)^{2} I a^{2}\right\} U \\
& +\left\{(\nu-2)(\nu-3)^{3}+(\nu-2)(\nu-3)(2 \nu-5) J a^{2}\right\} \nabla U .
\end{aligned}
$$

En représentant par $i, j, k, l, m$, les coefficients différentiels du quatrième ordre de $U$, on a

$$
\begin{aligned}
& I=i k m-i l^{2}-j m^{2}-k^{3}+2 j k l, \\
& J=4 j l-3 k^{2}-m i,
\end{aligned}
$$

de manière que $I, J$ sont des fonctions de $x, y$ des ordres $3(\nu-4)$ et $2(\nu-4)$ respectivement."

Ce qu'il y a de remarquable, c'est la forme de ces deux quantités $I, J$. On voit d'abord que la fonction $I$ est la déterminante formée avec les termes

$$
\begin{array}{lll}
i, & j, & k \\
j, & k, & l \\
k, & l, & m .
\end{array}
$$

Mais de plus les deux fonctions $I, J$ ont la propriété suivante. $\mathrm{Si}$ l'on imagine une fonction du quatrième ordre

$$
l \xi^{4}+4 j \xi^{3} \eta+6 k \xi^{2} \eta^{2}+4 l \xi \eta^{3}+m \eta^{4},
$$

transformée en

$$
l^{\prime} \xi^{\prime 4}+4 j^{\prime} \xi^{\prime 3} \eta^{\prime}+6 k^{\prime} \xi^{\prime 2} \eta^{\prime 2}+4 l^{\prime} \xi^{\prime} \eta^{\prime 3}+m^{\prime} \eta^{\prime 4}
$$

au moyen de

$$
\begin{aligned}
& \xi=\lambda \xi^{\prime}+\mu \eta^{\prime}, \\
& \eta=\lambda^{\prime} \xi^{\prime}+\mu^{\prime} \eta^{\prime},
\end{aligned}
$$

en représentant par $I^{\prime}, J^{\prime}$, les mêmes fonctions de $i^{\prime}, j^{\prime}, k^{\prime}, l^{\prime}, m^{\prime}$, on a

$$
J^{\prime}=\left(\lambda \mu^{\prime}-\lambda^{\prime} \mu\right)^{6} . J, \quad I^{\prime}=\left(\lambda \mu^{\prime}-\lambda^{\prime} \mu\right)^{4} . I .
$$


L'on parviendrait, je crois, à des résultats plus simples en considérant une fonction $U$, homogène en $x, y$, et aussi en $x_{1}, y_{1}$, et en posant

$$
\nabla U=\frac{d^{2} V}{d x d x_{1}} \cdot \frac{d^{2} V}{d y d y_{1}}-\frac{d^{2} V}{d x d y_{1}} \cdot \frac{d^{2} V}{d y d x_{1}} .
$$

Par exemple, si $U$ est du second ordre en $x, y$ et aussi en $x_{1}, y_{1}$, de manière que

$$
\begin{aligned}
U= & x_{1}{ }^{2}\left(A x^{2}+2 B x y+C y^{2}\right) \\
& +2 x_{1} y_{1}\left(A^{\prime} x^{2}+2 B^{\prime} x y+C^{\prime} y^{2}\right) \\
& +y_{1}{ }^{2} \quad\left(A^{\prime \prime} x^{2}+2 B^{\prime \prime} x y+C^{\prime \prime} y^{2}\right)
\end{aligned}
$$

on a simplement

$$
\nabla \nabla U=\mathfrak{A} U,
$$

en représentant par $\mathfrak{A}$ la déterminante formée avec les coefficients

$$
\begin{array}{lll}
A, & B, & C ; \\
A^{\prime}, & B^{\prime}, & C^{\prime} ; \\
A^{\prime \prime}, & B^{\prime \prime}, & C^{\prime \prime} ;
\end{array}
$$

et multipliée par $2^{8}$. Mais il faudrait développer tout cela beaucoup plus loin.

P.S. Je ne sais pas si l'on a jamais discuté la question suivante, qui doit conduire, il me semble, à des résultats importants. Soient $L, M, L^{\prime}, M^{\prime}, U, V$ des fonctions de $x$. En éliminant cette variable entre les équations $L U+M V=0$ et $L^{\prime} U+M^{\prime} V=0$, et en représentant l'équation ainsi obtenue par $\left[L U+M V, L^{\prime} U+M^{\prime} V\right]=0$, et de même par $[U, V]=0$ l'équation obtenue en éliminant $x$ entre $U=0, V=0$, il est clair que $\left[L U+M U, L^{\prime} U+M^{\prime} V\right]$ contiendra $[U, V]$ comme facteur: quelles sont maintenant les propriétés de l'autre facteur qu'on peut écrire sous les trois

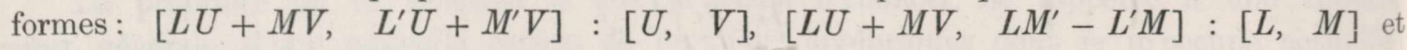
$\left.\left[L^{\prime} U+M^{\prime} V, L M^{\prime}-L^{\prime} M\right]:\left[L^{\prime}, M^{\prime}\right]\right) ?$ 